\title{
Malnutrition in Infants under 6 months: Is it Time to Change Recommendations?
}

\author{
Shivani Randev ${ }^{1}$ (D) \\ Received: 11 June 2020 / Accepted: 25 June 2020 / Published online: 11 July 2020 \\ (C) Dr. K C Chaudhuri Foundation 2020
}

Under-five mortality rate in India has been steadily decreasing but still the contribution of malnutrition to child deaths is quite high. Despite decades of nutritional interventions, out of the 1.04 million under-five children who died in India in the year 2017, almost 706,000 were malnourished [1]. Till date, most of the focus of nutritional mediations has been in children 6 to 59 mo of age for which WHO has given clear cut recommendations on identification and treatment. However, worldwide, some 8.5 million infants under 6 mo of age are malnourished, while the prevalence in India is estimated at $14.8 \%$ [2]. WHO recommendations exist for infants under $6 \mathrm{mo}$ also, but it is clearly mentioned that the level of evidence is 'very low quality', mostly because of a paucity of studies on evaluation of diagnostic criteria and therapeutic feeding options specifically in this age group [3]. As per WHO, Severe acute malnutrition (SAM) in an infant less than 6 mo of age is defined as weight-for-length $\mathrm{Z}$-score $(\mathrm{WLZ})<-3$ or bilateral pitting edema. The basis for this definition is mostly indirect using data from older infants. But can we really extrapolate information from older infants to this younger subgroup?

A substantial proportion of infants in this age group are those who were low birthweight due to prematurity or intrauterine growth retardation. They might be growing parallel to their centiles but will be classified as malnourished in a single time-point evaluation using current criteria. Also, several of these infants might be less than $45 \mathrm{~cm}$ in length and WLZ cannot be calculated for them using 2006 WHO growth charts. Apart from the ability to predict mortality, it is important for the anthropometric indicator to be easily measurable in community

Shivani Randev

shivanirandev@hotmail.com

1 Department of Pediatrics, Government Medical College and Hospital, Sector 32, Chandigarh 160030, India setting. There are practical problems in using a parameter like WLZ. Accurate length of a young infant might be difficult to measure by community health worker and easy access to infantometers is not always there.

Looking at the magnitude of problem, it follows that there is an urgent need to collect evidence specifically in this age group on which sound recommendations can be based.

The study by Kumar et al. published in this issue of the journal attempts to fill this gap in knowledge [4]. The authors have correlated a variety of anthropometric parameters and their combinations with risk of in-hospital mortality amongst 1813 hospitalized infants 1-6 mo of age. Weight-for-age z-scores (WAZ) less than -3 , WLZ less than -3 and Mid upper arm circumference (MUAC) $<10 \mathrm{~cm}$ were all significantly associated with high inhospital mortality, but WAZ was determined to have the best predictive ability after adjusting for age, sex and exclusive breast-feeding status. Using WAZ, prevalence of severe malnutrition was $39.9 \%$ amongst study population. Mortality was seen to be $11.1 \%$ in babies with $\mathrm{WAZ}<-3$, $9.9 \%$ in those with $\mathrm{WLZ}<-3$ compared to an overall mortality of $5.9 \%$ in the study population. Length could not be measured in $3.8 \%$ subjects and $2.3 \%$ of all in whom it was measured, it was less than $45 \mathrm{~cm}$ so WLZ could not be determined. The other WHO criterion of bilateral pitting edema was not seen in any patient. Though this study provides valuable information, there is no denying the fact that this is a hospital based study where study population might not be truly representative of the community, and mortality has only been assessed during the period of hospitalization with no post discharge follow-up available. For the inference to be truly valid, there is a need to conduct similar research in the community setting with a longer follow-up period.

In a previous study in Kenya, anthropometric assessment during hospitalization was used to predict mortality not only during hospital stay but also during post-discharge follow up till one year and $\mathrm{WAZ}<-3$ was determined to be the most 
sensitive predictor of mortality [5]. In another study by the same group in community setting, anthropometry at 2 mo of age was used to predict mortality within first year of life and the conclusion was that WAZ $<-3$ and MUAC $<11.5 \mathrm{~cm}$ were better predictors than WLZ $<-3$ [6].

The result of this new research has reinforced our doubts regarding the appropriateness of WLZ as the criterion for diagnosis of severe malnutrition in infants under 6 mo and highlighted the need to conduct more studies especially in community settings comparing various anthropometric parameters. There is also a need to collect evidence to address several other questions regarding malnutrition in this age group e.g., best therapeutic options, determining the anthropometric parameter to be used for monitoring response to therapy and formulating discharge criteria. Though undernutrition has consequences at any age, the effects are most detrimental during the period of rapid growth and neurodevelopment as in a young infant and if not addressed in time, many of them might become irreversible. So to break the passage of undernutrition from one generation to the next, it is imperative to target it as early as possible.

\section{Compliance with Ethical Standards}

\section{References}

1. India State-Level Disease Burden Initiative Collaborators. Nations within a nation: variations in epidemiological transition across the states of India, 1990-2016 in the Global Burden of Disease Study. Lancet. 2017;390:2437-60.

2. Choudhary TS, Srivastava A, Chowdhury R, et al. Severe wasting among Indian infants $<6$ months: findings from the National Family Health Survey 4. Matern Child Nutr. 2019;15:e12866.

3. World Health Organisation. Updates on the Management of Severe Acute Malnutrition in Infants and Children. Guideline. 2013. Available at: https://www.who.int/publications/i/item/ 9789241506328. Accessed 25th May 2020.

4. Kumar P, Meiyappan Y, Rogers E, et al. Outcomes of hospitalized infants aged one to six months in relation to different anthropometric indices - an observational cohort study. Indian J Pediatr. 2020. https://doi.org/10.1007/s12098-020-03236-9.

5. Mwangome M, Ngari M, Fegan G, et al. Diagnostic criteria for severe acute malnutrition among infants aged under 6 mo. Am J Clin Nutr. 2017;105:1415-23.

6. Mwangome M, Ngari M, Bwahere P, et al. Anthropometry at birth and at age of routine vaccination to predict mortality in the first year of life: a birth cohort study in BukinaFaso. PLoS One. 2019;14: e0213523.

Publisher's Note Springer Nature remains neutral with regard to jurisdictional claims in published maps and institutional affiliations.

Conflict of Interest None. 